J Neurol Neurosurg Psychiatry 2004;75(Suppl II):ii37-ii42. doi: 10.1136/jnnp.2004.040493

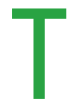
en to $50 \%$ of patients with systemic malignancy develop brain metastases during the course of their disease and metastases account for more than half of all brain tumours in adults. The major originating primary tumours are carcinomas typically arising from sites shown in fig 1 . The majority of patients have multiple metastases.

A long disease-free interval, with absent systemic metastatic disease and presentation of brain metastases of unknown primary, may require biopsy of the brain lesion if active treatment is appropriate. However, in the context of known metastatic disease histological confirmation is not usually necessary.

Up to one third of brain metastases are discovered on routine staging investigations, the majority presenting with varying clinical features. The diagnosis of brain tumours should be suspected in any cancer patient who develops any new neurological symptoms. Brain metastases present with headaches in $40-50 \%$ of patients, with increased frequency with multiple metastases or posterior fossa metastases. Seizures and behavioural symptoms are also common while, in comparison, focal neurological signs are rare as presenting symptoms.

In patients suspected to have brain metastases, contrast enhanced magnetic resonance imaging (MRI) is the best diagnostic test. However, current practice in the UK is to investigate initially with computed tomography (CT). If the CT scan shows multiple metastases then an MRI would not usually add any further information. However, in good performance status patients if a single metastasis is seen on CT imaging an MRI should be performed to exclude multiple metastases before more radical treatment (fig 2).

\title{
INITIAL MEDICAL MANAGEMENT OF BRAIN METASTASES
}

Initial medical management is with corticosteroids to treat peri-tumour oedema. Corticosteroids are effective for symptom control with the majority of patients improving in the first few days. Dexamethasone has been used since 1961 and acts in part by reducing the permeability of tumour capillaries. Potential advantages over other corticosteroids include reduced cognitive impairment and less mineralocorticoid activity, reducing the risk of fluid retention and hypokalaemia.

Conventionally the dose of dexamethasone is $16 \mathrm{mg}$ /day and, due to the long biological half-life of dexamethasone, there is no advantage in divided dosing over the 24 hour period. A small double blind randomised control study (RCT) showed that using $4 \mathrm{mg}, 8 \mathrm{mg}$, or $16 \mathrm{mg}$ of dexamethasone in 89 patients with brain metastases produced no difference in Karnofsky performance status after one week of treatment. ${ }^{1}$ Toxicity was dose dependent and more prevalent in patients receiving $16 \mathrm{mg} / 24$ hour dexamethasone. In longer term use of corticosteroids patients have increased risk of gastritis, hyperglycaemia, immunosuppression, insomnia, mood changes, oral candidiasis, proximal myopathy, and weight gain. Therefore the lowest dose of dexamethasone should be used for adequate symptom control to reduce the risk of corticosteroid complications.

Standard anticonvulsants are given if the patient presents with fits but there is no evidence that they are beneficial prophylactically. ${ }^{2}$ However, case reports have suggested that a combination of phenytoin and cranial irradiation is associated with a small increased risk of severe skin reactions including erythema multiforme and Stevens-Johnson syndrome.

Active treatment is only appropriate in patients who have a reasonable quality of life and a meaningful life expectancy.

See end of article for authors' affiliations

Correspondence to: Dr K N Franks, Cookridge Hospital, Leeds Teaching Hospitals NHS Trust, Hospital Lane, Leeds LSI6 6QB, UK; knfranks@hotmail.com

\section{ONCOLOGICAL TREATMENT OF PATIENTS WITH MULTIPLE BRAIN METASTASES Whole brain radiotherapy}

Whole brain radiotherapy (WBRT) remains the treatment of choice though the impact of WBRT on survival and quality of life compared to best supportive care has not been tested in randomised control trials. Current perception based on phase II studies is that WBRT achieves improvement in intracranial disease control with functional improvement in two thirds of patients. This relatively high response rate is from older studies and two recent papers have shown a much lower response 


\section{ii38}

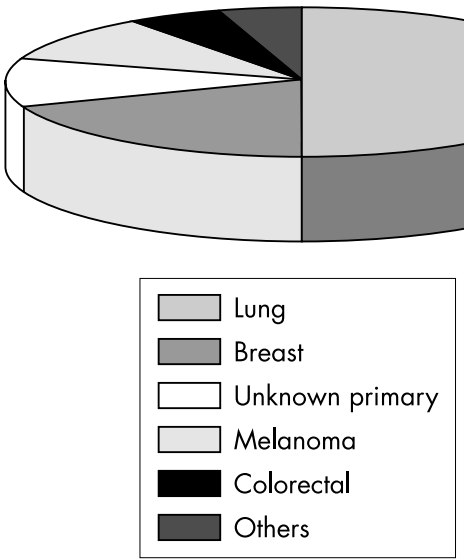

Figure 1 Common causes of brain metastases.

with less than a third of patients deriving benefit. However, the reduced response rate may only reflect a difference in comparing physician and patient questionnaire assessment.

In patients with two or more poor prognostic features whole brain radiotherapy may not provide effective palliation for the majority of these patients. Gaspar and colleagues performed a retrospective analysis of 1200 patients and suggested three classes of patients: class 1 with a Karnofsky performance status (KPS) $\geqslant 70,<65$ years of age with controlled primary and no extracranial metastases; class 3 KPS $<70$; class 2 all others. Class 1 patients had a median survival of 7.1 months compared with class 3 with median survival of 2.3 months. $^{3}$

WBRT can also have significant acute and late effects which should be taken into consideration when deciding whether to treat a patient palliatively. Acute effects include alopecia, skin erythema, cerebral oedema with transient worsening back to pre-radiotherapy neurological deficit, and very small risk of death. Late side effects include somnolence syndrome, neuroendocrine dysfunction, neurocognitive decline, and normal pressure hydrocephalus. Neurocognitive decline is an important cause of morbidity and there is currently a phase II study looking at the efficacy of methylphenidate (Ritalin), a pychostimulant used in the treatment of psychiatric conditions, as a treatment of neurocognitive effects post-radiotherapy.

Factors that predict for late effects are total dose, dose per fraction (higher dose per fraction results in greater late effects), pre-morbid condition, and extent of disease. Studies in which patients were given one week of radiotherapy versus, two, three, and four or five weeks of radiotherapy showed no survival difference and hence there is no consensus for the ideal dose and schedule.

In the UK the standard treatment is either $20 \mathrm{~Gy}$ in five fractions over one week or $30 \mathrm{~Gy}$ in 10 fractions over two weeks to the whole brain. Currently there is no evidence that in patients with multiple brain metastases any additional treatment to WBRT contributes further clinical benefit. However, trials with addition of biological agents are planned and may prove to act synergistically with radiotherapy.

\section{Systemic treatments}

Systemic treatments are being increasingly used in patients presenting with brain metastases. It would seem logical to use systemic agents as more than half of the patients treated
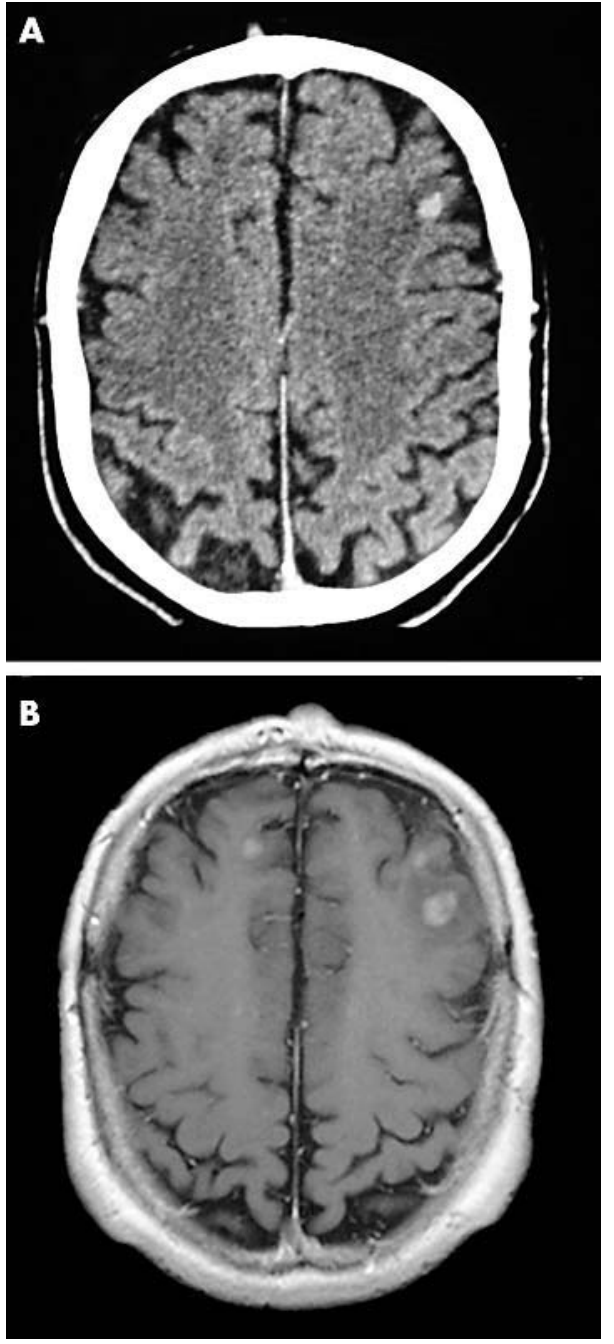

Figure 2 (A) Single metastasis visible on computed tomographic (CT) head scan. (B) Multiple metastases visible in the same patient on magnetic resonance imaging (MRI).

with surgery or radiotherapy for their brain metastases will die as a result of systemic disease. Chemotherapy has been used in a variety of tumour types with disappointing results. It had been thought that the blood-brain barrier prevented chemotherapy agents crossing into the cerebrospinal fluid, but chemotherapy responsiveness of enhancing brain metastases is similar to systemic disease metastases. Therefore chemotherapy is appropriate in patients with chemosensitive tumours such as small cell lung cancer, non-Hodgkin's lymphoma, and germ cell tumours. Chemotherapy has also produced responses in brain metastases from breast cancer and non-small cell lung cancer (NSCLC), though no RCTs have compared chemotherapy to WBRT.

Prognostic factors in patients with brain metastases

Performance status

Age

Presence of other metastatic disease

Status of primary disease 
Despite increasing numbers of studies examining the additional role of chemotherapy agents the potential clinically meaningful benefit is likely to be small. Chemotherapy may have a role in patients relapsed after WBRT and in patients with systemic disease and small asymptomatic brain metastases.

In patients with poor performance status (KPS $<70$ ), advanced non-cranial disease, and poor life expectancy, best supportive care is likely to be the best option with dexamethasone, at the lowest symptom controlling dose, and palliative care support.

\section{ONCOLOGICAL TREATMENT OF PATIENTS WITH SOLITARY BRAIN METASTASES}

Patients with radiologically proven solitary brain metastases should be considered for either primary surgical excision or radiosurgery. ${ }^{4}$

\section{Surgery}

Surgery is the primary treatment option in patients with an MRI verified surgically accessible solitary brain metastasis, providing the patient is of good performance status and has minimal or no evidence of extracranial disease.

Surgical removal of single brain metastasis followed by radiotherapy has been compared to radiotherapy alone in prospective randomised control trials. Patchell and colleagues randomised 48 patients to either biopsy of the lesion plus WBRT or complete resection of the metastasis plus WBRT. Both groups received 36 Gy in 12, 3 Gy fractions over 12 days. Recurrence at the site of the original metastasis was less frequent in the surgically resected group (20\% v 52\%; $\mathrm{p}<0.02$ ) and the overall survival was longer in the surgically resected group (median, 40 weeks $v 15$ weeks; $\mathrm{p}<0.01$ ). ${ }^{5} \mathrm{~A}$ second RCT of 63 patients performed in the Netherlands confirmed these results with a significant increase in overall survival in the surgically resected followed by WBRT compared with WBRT alone (10 months $v 6$ months; $\mathrm{p}=0.04)$. This was more pronounced in patients with stable extracranial disease (median overall survival 12 months $v 7$ months). They also measured functionally independent survival (FIS) which was also improved in the combined arm. However, they used a non-standard radiation dose with two fractions per day up to a total dose of $40 \mathrm{~Gy}$; patients who were randomised to radiotherapy alone did not have histological confirmation and MRI imaging was not performed to exclude multiple small metastases. ${ }^{6}$ A third larger RCT showed that there was no benefit from surgery plus radiotherapy compared with radiotherapy alone, though

Abbreviations

ASCO: American Society of Clinical Oncology
CNS: central nervous system
CSF: cerebrospinal fluid
CT: computerised tomography
GP: general practitioner
KPS: Karnofsky performance status
ICP: intracranial pressure
MRI: magnetic resonance imaging
NSCLC: non-small cell lung cancer
RCT: randomised control trial
SCC: spinal cord compression
WBRT: whole brain radiotherapy

extracranial metastases were an important predictor for overall survival. $^{7}$

Surgery cannot be performed in all patients with solitary brain metastases because of the proximity of lesions to salient areas of the brain and those areas that are not accessible.

\section{Stereotactic radiosurgery}

Stereotactic radiosurgery is a non-invasive alternative to surgery and should be considered in patients with tumours $\leqslant 3.5 \mathrm{~cm}$ in diameter. It delivers high dose radiation to a focal area in the brain by delivering multiple convergent beams. These beams can be produced by using a dedicated multisource cobalt unit (gamma knife), stereotactic multiple arc radiotherapy from a linear accelerator, or protons produced by cyclotron. Because of the degree of accuracy necessary to treat lesions with radiosurgery, special immobilisation devices to prevent patient movement and CT or MRI imaging with computer planning systems are required.

In uncontrolled studies the efficacy of stereotactic radiosurgery is similar to surgery, though no direct comparison RCT has been performed. It produces local control rates of $73-94 \%$ though it is associated with a significant incidence of radiation necrosis of $5-10 \%$.

Other side effects include dizziness, nausea, and new onset headache that can occur within the first two days following radiosurgery, which tends to be relatively mild. Fitting can occur within the first week following radiosurgery and is more common in patients whose lesions lie in the motor cortex. Late side effects include radiation necrosis and treatment induced cranial neuropathies.

The role of WBRT following radiosurgery or following complete resection of solitary brain metastases is unclear. An RCT is to commence in the UK shortly to attempt to answer this question.

\section{SPINAL METASTASES}

Spinal metastases are a common consequence of malignant disease and approximately $10 \%$ of patients with cancer will develop spinal cord compression (SCC).

Bony spinal metastases can arise from any primary malignancy but occur commonly from prostate, breast, and lung cancer. Other less common causes are renal cell carcinoma, plasmacytoma/multiple myeloma, and nonHodgkin's lymphoma.

Spinal metastases without neurological compromise are more common than SCC though $20 \%$ of patients with spinal metastases will develop SCC. One fifth of cases of SCC present in patients without a known primary site.

Two thirds of SCC are in the thoracic region because of the narrower spinal canal. SCC presents with pain in over $80 \%$ of patients which often develops over 7-15 weeks before the onset of neurological symptoms.

The pain can be localised or radicular, it can be exacerbated by lying down and usually progressively worsens over a period of weeks. Motor weakness is present in $60-85 \%$ of patients with SCC, usually producing bilateral leg weakness.

Sensory signs are less common than motor weakness, often with a sensory level, but can occur in a radicular distribution, with ascending numbness or paresthesia. Bowel and bladder disturbances are late features but may develop in up to $50 \%$ of patients.

Neither the site of pain nor the sensory level often correlate with the actual level of cord compression. Unfortunately, 
because of delay in presentation and diagnosis malignant SCC is a significant cause of morbidity and mortality.

Therefore clinicians need to be aware of the possibility of SCC to aid early diagnosis and urgently arrange appropriate investigations. In a prospective study, most patients reported early symptoms to their general practitioner (GP), though the diagnosis was delayed for approximately two months from the GP visit. At diagnosis $82 \%$ of patients were unable to walk unaided. ${ }^{8}$

In patients with a known diagnosis of cancer, SCC should be suspected in patients with severe back or radicular pain. The investigation of choice is an MRI scan of the spine, as plain $x$ ray and isotope bone scans are inadequate for diagnosis and predicting the level of compression.

Once a diagnosis is suspected the patient should be commenced on steroids, usually dexamethasone $16 \mathrm{mg} / 24$ hours though higher doses, typically $96 \mathrm{mg} / 24$ hours, have been used. However, there is only one RCT trial of dexamethasone versus no steroids in patients treated with radiotherapy. ${ }^{9}$ This showed an improvement in gait function in the dexamethasone arm $(81 \% v 63 \%)$ and at six months a significant improvement in those patients still ambulatory (59\% v 33\%) in the dexamethasone arm.

Treatment with high dose steroids ( $96 \mathrm{mg} / 24$ hours) may be slightly more effective, but have a higher incidence of side effects.

\section{Surgery}

Surgery for spinal metastases can be considered and, with advances in surgical techniques, symptomatic and functional outcomes have improved. Traditionally a posterior decompression via laminectomy was the surgical technique in a patient with symptomatic SCC. However, the majority of metastatic deposits are in the anterior part of the vertebral body and this approach does not give neurological improvement if the anterior body of the vertebra has collapsed.

In view of the poor outcome from laminectomy, more radical surgical approaches are now used with the aim of complete excision of the metastases and immediate decompression of the spinal canal. This can be done via a retroperitoneal approach or thoracotomy depending on the site of metastasis, providing extensive exposure for complete resection and enabling anterior stabilisation. Bone grafting or the use of instrumentation and methylmethacrylate are used to achieve stabilisation. This has been reported in small retrospective studies to show an advantage over radiotherapy alone. In a recent abstract presented at the annual meeting of the American Society of Clinical Oncology (ASCO) in 2003, patients were randomised to radiotherapy alone or surgery followed by radiotherapy. The trial was stopped prematurely when planned interim analysis at 50\% accrual showed a significant difference in the number of days the patient remained ambulatory in the surgery following radiotherapy $\operatorname{arm}(126 v 35$ days; $\mathrm{p}=0.005)$. There were also improvements in levels of continence, daily steroid dose, and opiate use for pain control. The difference in overall survival between the two groups was not significant though there was a trend towards better survival in the surgical arm. Crossover from the radiotherapy arm to surgery was allowed in the trial if there was progressive weakness despite radiotherapy. Twenty per cent of patients who were nonambulatory after radiotherapy received surgery. Although there was a higher surgical complication rate $(40 \%$ compared with $12 \%$ in surgery initially), three of the 10 patients were ambulatory post-surgery.

The trial excluded patients with haematological and germ cell malignancies, both being chemotherapy and radiotherapy sensitive and primary spinal tumours.

This trial suggested that patients who received surgery followed by radiotherapy had better functional and symptomatic outcomes and surgery should be considered before radiotherapy for suitable patients. ${ }^{10}$

\section{Radiotherapy}

Radiotherapy is the current standard treatment for patients with metastatic spinal cord compression. Unfortunately, multiple levels of compression occur in approximately one third of patients with metastatic SCC, and the poor prognosis of many patients (mean survival 4-6 months) excludes a surgical option.

Radiotherapy is typically given from a posterior field to the affected vertebra(e), encompassing one vertebral body above and below to account for daily variations in patient setup. Doses used range from $8 \mathrm{~Gy}$ in a single fraction to up to 30 40 Gy over 3-4 weeks.

Although there are no randomised control trials comparing dose regimens, there is some evidence that lower doses may be as effective. The most commonly used regimen in the UK is $20 \mathrm{~Gy}$ in five fractions. Radiotherapy is most effective for improving pain and this is achieved in the majority of patients. Functional outcome is determined by the pretreatment neurological status of the patient. Radiotherapy preserves neurological function in the majority of patients and $80-100 \%$ of patients treated when still walking remain ambulatory. In contrast, only a minority with initial inability to walk improve in function enough to return to walking unaided, though this fraction improves in more radiosensitive tumours such lymphoma and myeloma.

The duration of symptoms also influences post-treatment function. The longer the duration of symptoms the better the result from radiotherapy.

\section{Chemotherapy}

Chemotherapy is not routinely used in the treatment of malignant SCC. It may be appropriate for chemosensitive tumours and has been used to good effect in germ cell tumours and lymphoma, and can be considered if surgery and radiotherapy are contraindicated. In highly chemosensitive tumours a response may be seen in days though in less sensitive tumours a response may take weeks, potentially allowing time for further damage to occur and reducing functional outcome.

Other treatments include embolisation, particularly in vascular tumours-for example, renal cell cancer and

Indications for consideration of surgery for
spinal metastases
- Life expectancy greater than three months
- Single site of compression
- Not completely paraplegic or paraplegia less than 12-24
hours
- Deterioration despite radiotherapy
- Bony deformity
Radio-resistant tumours


Spinal metastases: key points

High index of suspicion in cancer patients with severe back pain and neurological symptoms

- Early diagnosis and prompt investigations

- Consider surgery first in appropriate patients

- Early referral for treatment- "if the patient walks in they will walk out"

stereotactic radiosurgery to the spine. Percutaneous osteoplasty using CT guidance can be useful in pain from spinal metastases but is not used to treat the symptoms of spinal cord compression.

These techniques are not freely available in the UK and have not been compared to standard treatment in RCTs.

\section{MENINGEAL DISEASE (LEPTOMENINGEAL,} CARCINOMATOSIS MENINGITIS, CSF METASTASES)

Meningeal disease occurs in $5 \%$ of patients with cancer. Usually these patients have advanced disease at other sites and $50 \%$ have had brain metastases treated previously.

It is becoming increasingly common in non-haematological cancers, which may be as a consequence of improved diagnosis or with better systemic treatments leading to later relapse in sanctuary sites.

Meningeal disease caused by non-haematological tumours is associated with a median survival time of only three months. Treatment is often ineffective for a number of reasons; the main difficulty is delivering chemotherapy agents to the malignant cells in the central nervous system (CNS), and as meningeal disease presents as a late complication of malignant disease the general prognosis is poor.

The types of clinical features can be divided into three subgroups: (1) cerebral (cognitive impairment, headache, nausea and vomiting, and ataxia); (2) cranial neuropathies; and (3) spinal (back pain, radiculopathies).

These particular symptoms are produced by the tendency of malignant cells in the cerebrospinal fluid (CSF) to congregate in specific sites:

- base of skull producing cranial neuropathies, obstruction of CSF flow, and raised intracranial pressure (ICP)

- base of spine producing back pain, leg weakness, radiculopathies, bowel/bladder disturbance.

These areas are the most commonly identified radiologically and at postmortem examination, and is possibly caused by the effect of gravity and slow flow of CSF.

The gold standard for diagnosis is the identification of malignant cells in the CSF. Other typical CSF findings of meningeal disease are raised opening pressure, protein, lymphocytes, and reduced glucose. These findings are not diagnostic or specific but a completely normal CSF analysis is uncommon $(<10 \%)$ in diagnosed cases.

CSF cytology is a highly specific but non-sensitive test for the diagnosis of meningeal disease. The sensitivity can be increased by repeated CSF sampling. In one retrospective analysis of 49 patients diagnosed with meningeal disease, the first CSF sample was positive in $50 \%$ of patients, which increased to $90 \%$ after three separate samples. ${ }^{11}$ Similar findings have been found in other studies and it has been suggested that by increasing the volume of CSF removed, having immediate analysis, and repeat samples if previously negative, you are able can increase the sensitivity.
Common causes of meningeal metastases

- Haematological malignancies

- Lung cancer

- Breast cancer

Melanoma

CSF can also be analysed for tumour markers such as $\alpha$ fetoprotein, $\beta$ HCG, carcinoembryonic antigen (CEA), and Ca 15-3, though at present the sensitivity remains low. However, a raised CSF tumour marker in comparison to serum values strongly suggests the presence of meningeal disease.

MRI can provide definitive evidence of meningeal disease though its sensitivity and specificity are yet to be established.

Treatment includes radiotherapy, and intrathecal and systemic chemotherapy.

\section{Radiotherapy}

Radiotherapy can be effective at specific sites of meningeal disease and provides more rapid relief of symptoms.

Radiotherapy should be considered in patients with localisable lesions or those with symptomatic identifiable bulk disease on imaging. It is commonly used to treat specific problematic areas rather than the whole CNS. Craniospinal radiotherapy is not used in the palliation of meningeal disease as it does not eradicate the disease and has a high incidence of side effects, particularly myelosuppression.

The side effects of localised radiotherapy depend on the site irradiated. Acute side effects are usually minimal and short lived, and long term side effects are rare because of the general prognosis of the disease.

\section{Intrathecal chemotherapy}

Intrathecal chemotherapy has been the standard treatment for meningeal disease.

However, it has many limitations: (1) to deliver intrathecal chemotherapy the patient has to have either repeated lumbar punctures or the neurosurgical insertion of a reservoir and ventricular catheter. In the palliative setting both forms of delivery can have significant patient morbidity and effect quality of life; (2) currently only a small number of agents can be used which mainly have an effect against haematological malignancies rather than solid tumours; (3) when given intrathecally it relies on adequate CSF flow to deliver the chemotherapy agent into the subarachnoid space. However, intrathecal radionucleotide scans show that CSF flow is impaired in the majority of patients with meningeal metastases; (4) intrathecal chemotherapy does not eradicate bulk disease.

Methotrexate is the principle drug used in the treatment of both non-haematological malignancies and haematological malignancies. Intrathecal methotrexate can cause leucoencephalopathy with a significant increased risk in those patients receiving concurrent radiotherapy. Cytarabine and thiotepa can be given intrathecally and are used principally in haematological malignancies. Thiotepa has the advantage of being able to be given in conjunction with radiotherapy and in those patients with methotrexate induced leucoencephalopathy. Cytarabine is less effective in solid malignancies but is produced in sustained release form with a much longer half life, thereby reducing the frequency of injections. 


\section{Systemic chemotherapy}

Systemic chemotherapy has the theoretical advantage of treating both CNS and systemic disease. It also spares the patient either repeat lumbar punctures or the insertion of a ventricular reservoir. However, patients may have developed meningeal disease despite systemic chemotherapy, presumably due to the lack of chemotherapy penetrance into the CSF. Certain chemotherapy agents are lipid soluble such as thiotepa and therefore can cross the blood-brain barrier more efficiently.

Another strategy is to give high doses of agents to attain good CSF concentrations. High dose methotrexate has been used and can give CSF concentrations similar to intrathecal methotrexate, but requires prolonged hospital admission for intravenous bicarbonate, hydration, and folinic acid rescue. Other agents such as cytarabine can achieve CSF penetration at high doses but increase the risk of significant toxicity.

Unfortunately, thiotepa, cytarabine, and methotrexate are not particularly effective in the treatment for solid, nonhaematological malignancies, and therefore their role is limited.

Meningeal metastases disrupt the blood-brain barrier allowing intravenous chemotherapy agents to penetrate more effectively into the CSF. Theoretically, this would suggest that standard chemotherapy agents used in the treatment of metastatic disease would have an effect on meningeal deposits. In reality the effective penetration of systemic chemotherapy to meningeal deposits remains poor.

At present the role of systemic chemotherapy in treating meningeal disease alone has not been evaluated, though is widely used where there is co-existent meningeal and other sites of metastatic disease.

\section{Surgery}

The role of surgery is very limited in meningeal disease. It is principally used for the insertion of ventricular catheters/ reservoirs and ventriculoperitoneal shunts for the treatment of symptomatic hydrocephalus.

In practice, combinations of radiotherapy, and intrathecal and intravenous chemotherapy are used in the symptomatic management of leptomeningeal disease. Combination treatments in non-haematological malignancies do not appear to increase the response rates or overall survival but have a higher incidence of treatment related complications. Acute complications are related to insertion of the drain, injection of the intrathecal chemotherapy, and CNS infections. However, the risk of delayed leucoencephalopathy is increased when radiotherapy and intrathecal chemotherapy are combined.

Therefore evaluation of a patient's age, performance status, non-CNS systemic disease, and prognosis is required before deciding on therapeutic options. All the treatments can impact on quality of life and have significant complications, particularly intrathecal chemotherapy.
In haematological malignancies complete remission can be achieved by combinations of radiotherapy and intrathecal and intravenous chemotherapy. Symptomatic bulk disease should be treated by focused radiotherapy. In non-haematological malignancies the aim of treatment is improvement in overall survival and palliation. More aggressive treatments should be considered in patients with good performance status, well controlled non-CNS disease, and breast cancer.

\section{CONCLUSION}

Brain, spinal, and meningeal metastases are a common complication of cancer. Early diagnosis is important, especially in spinal cord compression, to obtain the best outcome from treatment. Treatment can often involve a combination of therapeutic modalities and therefore multidisciplinary assessment is required.

Careful consideration of the patient's performance status, prognosis, and wishes are necessary before embarking on treatment. Although some treatments can be curative the majority are palliative and therefore the patient's quality of life is of key importance.

\section{Authors' affiliations}

G Gerrard, K N Franks, Cookridge Hospital, Leeds Teaching Hospitals NHS Trust, Leeds, UK

\section{REFERENCES}

1 Vecht CJ, Hovestadt A, Verbiest HB, et al. Dose-effect relationship of dexamethasone on Karnofsky performance in metastatic brain tumors: a randomized study of doses of 4,8 , and $16 \mathrm{mg}$ per day. Neurology 1994:44:675.

2 Forsyth PA, Weaver S, Fulton D, et al. Prophylactic anticonvulsants in patients with brain tumour. Can J Neurol Sci 2003;30:106-12.

3 Gaspar L, Scott C, Rotman M, et al. Recursive partitioning analysis (RPA) of prognostic factors in three radiation therapy oncology group (RTOG) brain metastases trials. Int J Radiat Oncol Biol Phys 1997;37:745-51.

4 Hoskin PJ, Brada M. Second workshop on palliative radiotherapy and symptom control. Radiotherapy for brain metastases. Clin Oncol (R Coll Radiol) 2001;13:91-4.

5 Patchell RA, Tibbs PA, Walsh JW, et al. A randomized trial of surgery in the treatment of single metastases to the brain. N Engl J Med 1990;322:494-500.

6 Vecht CJ, Haaxma-Reiche H, Noordijk EM, et al. Treatment of single brain metastasis: radiotherapy alone or combined with neurosurgery? Ann Neurol 1993;33:583-90.

7 Mintz AH, Kestle J, Rathbone MP, et al. A randomized trial to assess the efficacy of surgery in addition to radiotherapy in patients with a single cerebral metastasis. Cancer 1996;78:1470-6.

8 Levack P, Graham J, Collie D, et al. Don't wait for a sensory level-listen to the symptoms: a prospective audit of the delays in diagnosis of malignant cord compression. Clin Oncol 2002; 14:472-80.

9 Sorensen S, Helweg-Larsen S, Mouridsen $\mathrm{H}$, et al. Effect of high-dose dexamethasone in carcinomatous metastatic spinal cord compression treated with radiotherapy: a randomised trial. Eur J Cancer 1994;30A:22-7.

10 Regine WF, Tibbs PA, Young A, et al. Metastatic spinal cord compression: a randomized trial of direct decompressive surgical resection plus radiotherapy vs. radiotherapy alone. Int J Radiat Oncol Biol Phys 2003;57(2 suppl):S125.

11 Wasserstrom WR, Glass JP, Posner JB. Diagnosis and treatment of leptomeningeal metastases from solid tumours. Experience with 90 patients. Cancer 1982;49:759-72. 